Objectives: To explore latent patterns of clinical juvenile arthritis disease activity scores (cJADAS) following a diagnosis of JIA.

Methods: CYP with JIA were selected if enrolled in the Childhood Arthritis Prospective Study (CAPS), a UK multicentre inception cohort, before January 2015. cJADAS10 scores were calculated based on components (active joint count up to 10 , physician global, patient/parent global) collected at diagnosis, six months, one year and then annually to three years. CYP were excluded if no cJADAS10 scores were available within this time frame.

Group-based trajectory models were constructed to model latent groups of cJADAS10 scores. Linear, quadratic and cubic polynomials were tested, with one to six trajectories tested within each polynomial group. An optimal model within each polynomial group was selected using Bayesian Information Criteria. The final model was then selected from this shortlist based on model parsimony and clinical plausibility.

Results: Of 1183 CYP selected, the majority were female $(65 \%)$ and of white ethnicity $(90 \%)$ with oligoarticular JIA the most common JIA category (45\%). The optimal model identified five cJADAS10 quadratic trajectories (Figure 1): Low-low (59\%, initial cJADAS10 median: 6.1), moderate-low (16\%, initial cJADAS10 median: 11.5) and three groups with high disease activity at initial presentation (initial median CJADAS10: 17.7 to 19.1). A high-low group experienced the greatest improvement (15\%, median improvement 17.2 (IQR 13.7 to 20.1)), and a high-moderate group lesser improvement (5\%, median improvement 7.3 (IQR 0.8 to 9.0)). A final high-low-high group experienced improvement to one year followed by disease relapse (5\%). Conclusion: Disease activity in CYP with JIA does not improve in a uniform manner following initial presentation to paediatric rheumatology. Five latent trajectory groups have been identified, with three of these displaying different patterns following initial high disease activity at diagnosis. Identifying distinguishing characteristics for each group may aid the stratification of different treatment strategies to facilitate personalised medicine in JIA.

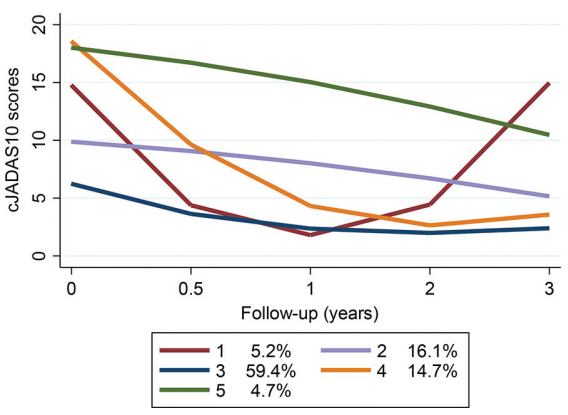

Figure 1. Latent trajectories of CJADAS10 scores in young people with JIA over the first three years following diagnosis

Acknowledgement: On behalf of CAPS and CLUSTER Disclosure of Interests: Stephanie Shoop-Worrall Grant/research support from: Your Rheum is funded by the Arthritis Research UK grant 20164, via the Centre for Adolescent Rheumatology at UCL. The event described here was funded by Eli Lilly., Kimme Hyrich Grant/research support from: Grants to institution: BMS, Pfizer, UCB, Lucy Wedderburn: None declared, Wendy Thomson: None declared, Nophar Geifman: None declared DOI: 10.1136/annrheumdis-2019-eular.2412

\section{FRI0547 DEVELOPMENT AND INITIAL VALIDATION OF THE SYSTEMIC JADAS, A NEW COMPOSITE DISEASE ACTIVITY SCORE FOR SYSTEMIC JUVENILE IDIOPATHIC ARTHRITIS}

Jessica Tibaldi ${ }^{1}$, Yasser El Miedany ${ }^{2}$, Priyankar $\mathrm{Pal}^{3}$, Soamarat Vilaiyuk ${ }^{4}$, Raju Khubchandani ${ }^{5}$, Claudia Bracaglia ${ }^{6}$, Tapas K. Sabui ${ }^{3}$, Sujata Sawhney ${ }^{7}$, Riccardo Russo ${ }^{8}$, Flávio R. Sztajnbok ${ }^{9}$, Rolando Cimaz $^{10}$, Francesca Minoia ${ }^{11}$, Motasem O. Alsuweiti ${ }^{12}$, Ekaterina Alexeeva ${ }^{13}$, Mikhail Kostik ${ }^{14}$, Maria Cristina Maggio ${ }^{15}$, Nicolino Ruperto ${ }^{1}$, Alessandro Consolaro ${ }^{1}$, Angelo Ravelli ${ }^{1}{ }^{1}$ Ist G.Gaslini, Genoa, Italy, ${ }^{2}$ Ped Rheum, Cairo, Egypt, ${ }^{3}$ Ped Rheum, Kolkata, India; ${ }^{4}$ Ped Rheum, Bangkok, Thailand; ${ }^{5}$ Ped Rheum, Mumbai, India; ${ }^{6}$ Ped Rheum, Rome, Italy; ${ }^{7}$ Ped Rheum, New Dheli, India; ${ }^{8}$ Ped Rheum, Buenos Aires, Argentina; ${ }^{9}$ Ped Rheum, Rio de Janeiro, Brazil; ${ }^{10}$ Ped Rheum, Florence, Italy; ${ }^{11}$ Ped Rheum, Milan, Italy; ${ }^{12}$ Ped Rheum, Amman, Jordan; ${ }^{13}$ Ped Rheum, Moscow, Russian Federation; ${ }^{14}$ Ped Rheum, Saint Petersburg, Russian Federation; ${ }^{15}$ Ped Rheum, Palermo, Italy

Background: Juvenile Arthritis Disease Activity Score (JADAS) has gained increasing popularity for the measurement of the level of disease activity in patients with juvenile idiopathic arthritis (JIA). However, so far the JADAS has been validated only in children with the non-systemic categories of JIA.

Objectives: To develop and validate the systemic JADAS (sJADAS), a new version of the JADAS specific to systemic JIA (SJIA).

Methods: The SJADAS is made up by adding a fifth item, named Systemic Manifestation Score (SMS), to the four items included in the original tool (physician global assessment of disease activity, parent/patient global assessment of well-being, active joint count and erythrocyte sedimentation rate). The SMS ranges from 0 to 10 and is aimed to quantify the activity of systemic features. The sJADAS score ranges from 0 to 50. The validation sample included patients with definite and possible/ probable SJIA with active systemic manifestations, which should comprise fever, who were assessed at baseline and then at a subsequent visit, 2 weeks to 3 months after initial evaluation. Validation procedures included assessment of concurrent, construct and discriminant validity, internal consistency and responsiveness to clinical change.

Results: A total of 161 patients, $86.7 \% \quad(n=137)$ with definite sJIA and $13.3 \%(n=21)$ with possible/probable sJIA, assessed at disease onset $(n=90 ; 56.6 \%)$ or at time of a disease flare $(n=69 ; 43.4 \%)$, were enrolled at 57 centers in 10 countries from February 2017 to December 2018. Median age at disease onset was 5.0 years (interquartile range, IQR 2.8

8) and median age at study entry was 6.9 years (IQR 3.8-10.8). Median disease duration from onset to study entry was 0.2 years (IQR 0.1-1.9). The median SJADAS at baseline visit was 28.2 (IQR 22.835.0). The SJADAS correlated strongly with JADAS10 $\left(r_{s}=0.98\right)$ and clinical JADAS10 (CJADAS10) $\left(r_{s}=0.91\right) ;$ moderately with functional ability scales (JAFS and CHAQ) $\left(r_{s}=0.69 ; r_{s}=0.62\right)$ and total score $\left(r_{s}=0.57\right)$ and physical $\left(r_{s}=0.60\right)$ and psychosocial $\left(r_{s}=0.40\right)$ subscale scores of the health-related quality of life tool (PRQL) and with pain VAS $\left(r_{s}=0.57\right)$ and mildly with the CRP $\left(r_{s}=0.42\right)$. The sJADAS discriminated well between patients with or without morning stiffness $(p<0.0001)$, with different levels of disease activity defined subjectively by the physician $(p<0.0001)$ and with different degrees of pain $(p<0.0001)$. The internal consistency was good (Cronbach's alpha=0.64) and comparable to that of JADAS10 (Cronbach's alpha=0.59,) and CJADAS10 (Cronbach's alpha=0.63). Responsiveness to change, measured on all patients $(\mathrm{SRM}=2.23)$ and on patients classified as improved at second visit $(\mathrm{SRM}=2.41)$, was strong and superior to that of JADAS10 (SRM $=1.92$ and 2.14 , respectively).

Conclusion: The SJADAS was found to be a valid instrument for the assessment of disease activity in SJIA. This composite score is feasible and easily applicable in standard clinical practice, which should result in its widespread acceptance and use. The strong responsiveness to clinical change over time indicates that the SJADAS is suitable to assess therapeutic response in SJIA clinical trials.

Disclosure of Interests: : Jessica Tibaldi: None declared, Yasser El Miedany: None declared, Priyankar Pal : None declared, Soamarat Vilaiyuk: None declared, Raju Khubchandani: None declared, Claudia Bracaglia: None declared, Tapas K Sabui : None declared, Sujata Sawhney: None declared, Riccardo Russo Speakers bureau: Novartis, Abbvie, Flávio R Sztajnbok: None declared, Rolando Cimaz: None declared, Francesca Minoia: None declared, Motasem O. Alsuweiti: None declared, Ekaterina Alexeeva: None declared, Mikhail Kostik: None declared, Maria Cristina Maggio: None declared, Nicolino Ruperto Grant/research support from: The Gaslini Hospital, where NR works as full-time public employee, has received contributions (> 10.000 USD each) from the following industries in the last 3 years: BMS, Eli-Lilly, GlaxoSmithKline, F Hoffmann-La Roche, Janssen, Novartis, Pfizer, Sobi. This funding has been reinvested for the research activities of the hospital in a fully independent manner without any commitment with third parties., Consultant for: Received honoraria for consultancies or speaker bureaus (< 10.000 USD each) from the following pharmaceutical companies in the past 3 years: Ablynx, AbbVie, Astrazeneca-Medimmune, Biogen, Boehringer, Bristol-Myers Squibb, Eli-Lilly, EMD Serono, GlaxoSmithKline, Hoffmann-La Roche, Janssen, Merck, Novartis, Pfizer, R-Pharma, SanofiServier, Sinergie, Sobi and Takeda., Speakers bureau: Received honoraria for consultancies or speaker bureaus (< 10.000 USD each) from the following pharmaceutical companies in the past 3 years: Ablynx, AbbVie, Astrazeneca-Medimmune Biogen, Boehringer, Bristol-Myers Squibb, Eli-Lilly, EMD Serono, GlaxoSmithKline, Hoffmann-La Roche, Janssen, Merck, Novartis, Pfizer, RPharma, SanofiServier, Sinergie, Sobi and Takeda., Alessandro Consolaro Grant/research support from: AbbVie, Pfizer, Angelo Ravelli Grant/research support from: Angelini, AbbVie, Bristol-Myers Squibb, Johnson \& Johnson, Novartis, Pfizer, Reckitt Benkiser, and Roche, Consultant for: Angelini, AbbVie, Bristol-Myers Squibb, Johnson \& Johnson, Novartis, Pfizer, Reckitt Benkiser, and Roche, Speakers bureau: Angelini, AbbVie, Bristol-Myers 
Squibb, Johnson \& Johnson, Novartis, Pfizer, Reckitt Benkiser, and Roche

DOI: 10.1136/annrheumdis-2019-eular.7415

\section{FRI0548 EFFICACY AND SAFETY OF TOCILIZUMAB IN SYSTEMIC AND POLYARTICULAR JUVENILE IDIOPATHIC ARTHRITIS - DATA OF THE BIKER REGISTRY}

Ariane Klein ${ }^{1}$, Gerd Ganser ${ }^{2}$, Ralf Trauzeddel ${ }^{2}$, Kisten Minden ${ }^{2}$,

Christoph Rietschel ${ }^{2}$, Jasmin Kuemmerle-Deschner ${ }^{2}$, Gerd Horneff ${ }^{1} .{ }^{1} B I K E R-$

Registry, Sankt Augustin, Germany; ${ }^{1}$ BIKER-Registry, Sankt Augustin, Germany

Background: Since 2011 Tocilizumab is approved for systemic juvenile idiopathic arthritis (JIA) and since 2013 for polyarticular JIA

Objectives: To describe efficacy and safety of Tocilizumab in clinical practice in polyarticular (pJIA) and systemic JIA (sJIA) patients using the German Biologics registry (BiKeR)

Methods: Baseline demographics and disease activity parameters have been documented. Efficacy was determined using the JADAS10 prospectively. Safety assessments were based on adverse events reports (AE) processed according to MedDRA

Results: Until October 1, 2018, 345 JIA patients treated with Tocilizumab were registered, representing 635 patient-years (PY) of observation. The cohort treated with Tocilizumab had experienced disease duration of 5.5 $+/-4.3$ years (mean+/-SD) for sJIA and 5.9+/-4.1 years for pJIA. sJIA/pJIA patients received pretreatment with MTX $72 \% / 96 \%$, Anakinra $23 \% / 1 \%$, Adalimumab $4 \% / 60 \%$, Etanercept $30 \% / 69 \%$, Canakinumab $2 \% / 0 \%$. Concomitantly, sJIA/pJIA patients received NSAIDs $64 \% / 57 \%$, systemic steroids $72 \% / 35 \%$, MTX $55 \% / 54 \%$, other DMARDS $4 \% / 8 \%$. At last follow-up, $63 \% / 60 \% / 51 \% / 43 \%$ of sJIA and $56 \% / 49 \% / 40 \% / 30 \%$ of pJIA patients reached JIAACR30/50/70/90 criteria. After 2 years of treatment JADAS remission was reached by $50 \% / 43 \%$ and JADAS minimal disease activity by $67 \% / 67 \%$.

586 AE were reported during exposure plus 90 days of observation. The rate was significantly higher in SJIA (104/100PY [95\% Cl 92-119]) than in pJIA patients (79 [79-90]; RR 1.3; $p=0.003) .75$ qualified as serious $A E$ (SAE) with a higher rate in SJIA (22[16-29] vs. 8 [5-12], RR 2.6; $p<0.001)$. The most frequent $A E$ in the $s J I A / p J I A$ cohort were grouped in the MedDRA SOC infections and infestations $(n=92 / 76)$. Compared to pJIA, rates were significantly higher in sJIA patients for blood and lymphatic (RR 2.4; $p=0.019$ ), immune system (RR 2.6; $p=0.04$ ), infections \& infestations (RR 1.7, $\mathrm{p}<0,001$ ) and nervous system disorder (RR 2.6, $p=0.012$ ) and lower for general and administration site (RR $0.4 ; p=0.023$ ). 169 patients (49\%) discontinued treatment, $17 \%$ due to remission, $22 \%$ due to lack of efficacy and $9 \%$ because of intolerance.

Conclusion: The analysis adds to the established safety profile of tocilizumab in paediatric patients with systemic \& polyJIA. Differences were noted between pJIA and SJIA cohorts, the latter with higher rates of total number of adverse events, serious $\mathrm{AE}$, infections and cytopenias, probably due to higher doses or shorter application intervals. No new safety signals specific to the paediatric population were identified in this large cohort of JIA patients.

\begin{tabular}{|c|c|c|c|}
\hline & sJIA (n=109) & $\begin{array}{c}\text { pJIA } \\
(n=239)\end{array}$ & $\mathrm{RR} / \mathrm{p}$ \\
\hline All AE (rate[95\%Cl]) & $\begin{array}{l}\text { 234(104[92- } \\
119])\end{array}$ & $\begin{array}{l}252(79[70- \\
90])\end{array}$ & $\begin{array}{l}0.76 / \\
0.003\end{array}$ \\
\hline SAE & $\begin{array}{l}49(22[16- \\
29])\end{array}$ & $26(8[5-12])$ & $\begin{array}{c}0.38 / \\
<0.001\end{array}$ \\
\hline blood \& lymphatic system ${ }^{1}$ & $20(9[6-14])$ & $12(4[2-7])$ & $\begin{array}{l}0.42 / \\
0.019\end{array}$ \\
\hline gastrointestinal disorders & $13(6[3-10])$ & $30(9[7-14])$ & n.s. \\
\hline general disorder $\&$ administration site condition ${ }^{2}$ & $9(4[2-8])$ & $\begin{array}{c}30(9(7- \\
14])\end{array}$ & $\begin{array}{l}2.4 / \\
0.023\end{array}$ \\
\hline immune system disorders $^{3}$ & $13(6[3-10])$ & $7(2[1-5])$ & $\begin{array}{l}0.38 / \\
0.040\end{array}$ \\
\hline infections and infestations ${ }^{4}$ & $\begin{array}{c}92 \text { (41[33- } \\
50])\end{array}$ & $\begin{array}{c}76(24[19- \\
30])\end{array}$ & $\begin{array}{c}0.59 / \\
<0,001\end{array}$ \\
\hline injury, poisoning and procedural complications & $7(3[1-7])$ & $10(3[2-6])$ & n.s. \\
\hline $\begin{array}{l}\text { neoplasms benign, malignant and unspecified (incl } \\
\text { cysts and polyps) }\end{array}$ & $1(0[0-3])$ & $2(1[0-3]$ & n.s. \\
\hline nervous system disorders ${ }^{5}$ & $20(9[6-14])$ & $11(3[2-6])$ & $\begin{array}{l}0,39 / \\
0.012\end{array}$ \\
\hline skin and subcutaneous tissue disorders & $11(5[3-9])$ & $11(3[2-6])$ & n.s. \\
\hline
\end{tabular}

MedDRA-SOC: Rates are outlined as number during exposure +90 days follow up, censored if an alternative biologic has been applied. 1 MAS, neutron-, thrombocytopenia; 2 nausea, aphthosis; 3 allergic reactions; 4 viral and bacterial infections; 5 seizures
REFERENCES:

[1] Horneff G, et al. Arthritis Res Ther. 2016 Nov 24;18(1):272

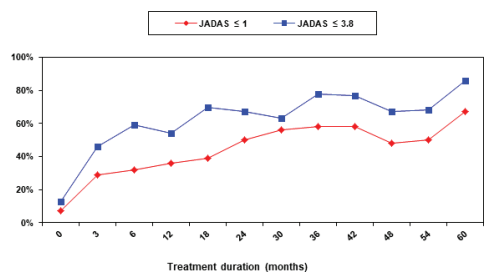

SJIA



polyJIA

Acknowledgement: BIKER and the documentation of the treatment with biologics is partially sponsored by Chugai and Roche, Germany

Disclosure of Interests: Ariane Klein: None declared, Gerd Ganser: None declared, Ralf Trauzeddel: None declared, Kisten Minden Grant/research support from: Pfizer, Abbvie, Christoph Rietschel: None declared, Jasmin Kuemmerle-Deschner Grant/research support from: Jasmin KuemmerleDeschner is an employee of University of Tuebingen, Germany, and received consultants/speakers fees from Novartis and SOBI pharmaceuticals and grant support from SOBI and Novartis., Consultant for: Jasmin Kuemmerle-Deschner is an employee of University of Tuebingen, Germany, and received consultants/speakers fees from Novartis and SOBI pharmaceuticals and grant support from $\mathrm{SOBI}$ and Novartis., Speakers bureau: Jasmin Kuemmerle-Deschner is an employee of University of Tuebingen, Germany, and received consultants/speakers fees from Novartis and $\mathrm{SOBI}$ pharmaceuticals and grant support from $\mathrm{SOBI}$ and Novartis., Gerd Horneff: None declared

DOI: 10.1136/annrheumdis-2019-eular.2109

\section{FRI0549 \\ SARILUMAB, A HUMAN MONOCLONAL ANTIBODY TO THE INTERLEUKIN-6 (IL-6) RECEPTOR, IN POLYARTICULAR-COURSE JUVENILE IDIOPATHIC ARTHRITIS (PCJIA): A 12-WEEK MULTINATIONAL OPEN-LABEL DOSE-FINDING STUDY}

Fabrizio De Benedetti ${ }^{1}$, Inmaculada Calvo Penadés ${ }^{2}$, Nadina E. Rubio Pérez ${ }^{3}$, Alexey Maschan ${ }^{4}$, Pierre Quartier ${ }^{5}$, Zbigniew Żuber ${ }^{6}$, Marina Stanislav 7 , Raul Barria ${ }^{8}$, Daniel Clemente Garulo ${ }^{9}$, Gabriel Vega Cornejo ${ }^{10}$, Nancy Liu ${ }^{11}$ Christine Xu ${ }^{11}$, Angeliki Giannelou ${ }^{12}$, Bolanle Akinlade ${ }^{12}$, Lydie Baret-Corme ${ }^{13}$, on behalf of DRI13925 investigators. 'Ospedale Pediatrico Bambino Gesù, Rome, Italy; ${ }^{2}$ Instituto de Investigación Sanitaria La Fe, Valencia, Spain; ${ }^{3}$ University Hospital Dr. José Eleuterio González, Monterrey, Mexico; ${ }^{4}$ Centre of Pediatric Hematology, Oncology and Immunology, Moscow, Russian Federation; ${ }^{5}$ Necker Hospital, Paris, France; ${ }^{6}$ Andrzej Frycz Modrzewski Krakow University, Krakow, Poland; ${ }^{7}$ VA Nasonova Research Rheumatology Institute, Moscow, Russian Federation; ${ }^{8}$ Bioreuma, Concepción, Chile; ${ }^{9}$ Hospital Infantil Universitario Niño Jesús, Madrid, Spain; ${ }^{10} \mathrm{CREA}$ de Guadalajara, Jalisco, Mexico; ${ }^{11}$ Sanofi, Bridgewater, NJ, United States of America; ${ }^{12}$ Regeneron, Tarrytown, NY, United States of America; ${ }^{13}$ Sanofi, Paris, France

Background: Sarilumab blocks IL-6 from binding to membrane and soluble IL-6 receptor-á. Sarilumab is approved for adults with rheumatoid arthritis (RA) and is being investigated in a Phase 2 trial (NCT02776735) 